\title{
The development of video game enjoyment in a role playing game
}

Wirth, Werner ; Ryffel, Fabian ; von Pape, Thilo ; Karnowski, Veronika

\begin{abstract}
This study examines the development of video game enjoyment over time. The results of a longitudinal study $(\mathrm{N}=62)$ show that enjoyment increases over several sessions. Moreover, results of a multilevel regression model indicate a causal link between the dependent variable video game enjoyment and the predictor variables exploratory behavior, spatial presence, competence, suspense and solution, and simulated experiences of life. These findings are important for video game research because they reveal the antecedents of video game enjoyment in a real-world longitudinal setting. Results are discussed in terms of the dynamics of video game enjoyment under real-world conditions.
\end{abstract}

DOI: https://doi.org/10.1089/cyber.2012.0159

Posted at the Zurich Open Repository and Archive, University of Zurich ZORA URL: https://doi.org/10.5167/uzh-83852

Journal Article

Published Version

Originally published at:

Wirth, Werner; Ryffel, Fabian; von Pape, Thilo; Karnowski, Veronika (2013). The development of video game enjoyment in a role playing game. Cyberpsychology, Behavior, and Social Networking, 16(4):260-264.

DOI: https://doi.org/10.1089/cyber.2012.0159 


\title{
The Development of Video Game Enjoyment in a Role Playing Game
}

\author{
Werner Wirth, PhD, Fabian Ryffel, MA, Thilo von Pape, $\mathrm{PhD}$, and Veronika Karnowski, $\mathrm{PhD}^{3}$
}

\begin{abstract}
This study examines the development of video game enjoyment over time. The results of a longitudinal study $(N=62)$ show that enjoyment increases over several sessions. Moreover, results of a multilevel regression model indicate a causal link between the dependent variable video game enjoyment and the predictor variables exploratory behavior, spatial presence, competence, suspense and solution, and simulated experiences of life. These findings are important for video game research because they reveal the antecedents of video game enjoyment in a real-world longitudinal setting. Results are discussed in terms of the dynamics of video game enjoyment under real-world conditions.
\end{abstract}

\section{Introduction}

$\mathrm{T}_{\mathrm{i}}$ HE PAST FEW years have witnessed a remarkable increase in interest in video game enjoyment among communication researchers. Researchers have described a wide array of components of video game enjoyment, including rather basic processes, such as, the experience of self-efficacy, as well as more complex processes, such as curiosity and exploration, ego-emotions, such as pride and joy, and processes of identification. ${ }^{1-5}$ Some of these components have been empirically verified on an individual basis in experimental studies; however, others have not yet been empirically proven. Moreover, knowledge about the interplay and development of these variables over time is sparse. However, as video games are mostly played over several sessions, the temporal component is critical to an understanding of video game enjoyment.

The present study examines the influence of several predictor variables that have been assumed to influence video game enjoyment, using a longitudinal design. In doing so, the results of previous laboratory studies were investigated in a field setting, thereby providing ecological validity.

\section{Concepts and Hypotheses}

In the following paragraph, the central theoretical assumptions of the present study are discussed. The variables used to predict enjoyment were identified in different experimental studies in the field of video game enjoyment research or in recent theoretical examinations.

\section{Exploratory behavior}

Modern role-playing video games provide elaborate and extensive virtual environments for players. Moreover, players have the opportunity to choose between different weapons, means of transportation (e.g., horses, cars, walking), and so on. Thus, players are offered many possibilities for acting in unknown terrains. For example, a player can move his or her avatar at various speeds in virtually any direction, or try out the effects of different weapons on various objects located in the fantasy environment. ${ }^{5}$ According to Vorderer et al., ${ }^{5}$ such exploratory behavior is enjoyable, as it is similar to the playful actions children perform when they explore what they can and cannot do with an object. Therefore, a causal link between exploratory behavior and enjoyment is expected.

Hypothesis 1a: The extent of exploratory behavior is positively associated with video game enjoyment.

Behr et al. ${ }^{1}$ assume that exploratory behavior is especially important for enjoyment in situations, where players are either overstrained or not sufficiently challenged, and therefore search for alternative possibilities to experience enjoyment. Although the authors assumed that exploration is especially a source of enjoyment under certain conditions, they were not able to demonstrate their hypothesis empirically. Nevertheless, it is highly plausible to assume that players who are not involved in completing game quests are more likely to draw enjoyment out of exploring the virtual environment than are players who are involved in game quests. Therefore, it is expected that the extent of enjoyment varies as a function

\footnotetext{
${ }^{1}$ Institute of Mass Communication and Media Research, University of Zurich, Zurich, Switzerland.

${ }^{2}$ Institute of Communication Research, University of Hohenheim, Stuttgart, Germany.

${ }^{3}$ Department of Communication Science and Media Research, Ludwig-Maximilians-University Munich, Munich, Germany.
} 
of both level of involvement in game tasks and extent of engagement in exploratory behavior. These observations suggest that players less involved in game quests would derive greater enjoyment out of exploring the game world than would players who were more involved in game quests.

Hypothesis 1b: Players less involved in game tasks show stronger relationships between exploratory behavior and video game enjoyment.

\section{Spatial presence}

Spatial presence is a binary state that can be conceptualized as perceived self-location in a mediated environment. ${ }^{6}$ Media experiences that evoke a feeling of being present in a mediated fictional surrounding have been suggested to be highly entertaining. ${ }^{7,8}$

Due to the fact that modern role-playing games feature high-quality graphics and sound, as well as a first-person viewpoint, all of which are important presence-inducing attributes, it is highly plausible that players in the present study enter a state of spatial presence. ${ }^{9}$ Therefore, a causal link between feelings of spatial presence and enjoyment is expected.

Hypothesis 2: The experience of spatial presence is positively associated with video game enjoyment.

\section{Competence}

A player's competence grows over the course of a video game. Players learn how to maneuver their characters, how to use weapons effectively, and how to negotiate confrontations with opponents. In short, players become more competent the longer they play the game; in addition, as a player's competence grows, the competitive elements in the game become more difficult. Games are mostly designed to operate at the outer expanding edge of a player's competence, remaining challenging, but doable. ${ }^{10}$ Therefore, players are constantly challenged, but can maintain a feeling of competency at every stage of their skill development through the course of a game. The feeling of competence is expected to be related to video game enjoyment because it is accompanied by positive emotions, such as pride and joy, as well as by increased feelings of self-esteem. ${ }^{4,11}$

Hypothesis 3: The feeling of competence is positively associated with video game enjoyment.

\section{Three mechanisms of video game enjoyment}

According to Klimmt, ${ }^{2}$ the video gaming process can be divided into three time units. Along these time units, the author conceptualizes video game enjoyment on three levels. On every level, there is a central mechanism that fosters video game enjoyment. These mechanisms will be discussed in the following sections.

Breaks in self-efficacy. The mechanism of self-efficacy takes place at the lowest level of Klimmt's ${ }^{2}$ model. From a temporal point of view, video game enjoyment on this level occurs by so-called input/output loops. Input/output loops consist of a situation presented on the screen (output), to which the gamer reacts with a certain input. This input leads to the presentation of a new situation, which requires a new input by the player. When the output immediately follows the input, and players experience themselves as the cause of the outcomes through various input/output loops; they enter a specific state of experience that Klimmt ${ }^{2}$ calls self-efficacy. The experience of self-efficacy is considered to be a fundamental aspect of video game enjoyment, although computer gamers are generally not aware of it under normal conditions. Self-efficacy as a component of video game enjoyment only becomes salient when it is missing; that is, in situations in which the computer game does not run fluently, such as when inputs by the player are delayed because of technical problems. ${ }^{2}$ Such interruptions of the experience of self-efficacy have been suggested to reduce enjoyment. ${ }^{3}$

Hypothesis 4: Breaks in the experience of self-efficacy are negatively associated with video game enjoyment.

Suspense and solution. On the second level of Klimmt's ${ }^{2}$ model, the mechanism of suspense and solution, is suggested to be related to video game enjoyment. This mechanism occurs during the second time unit, the so-called episode. Episodes consist of several input/output loops and confront video game players with a task or a challenge. Hence, episodes have a narrative content. Examples of episodes include overcoming an attacking enemy in a fighting game or outpacing an overtaking rival in a racing game. ${ }^{2}$

Episodes always evoke the necessity of action, and therefore put pressure on the player to perform. Since the negative ending of an episode can entail negative consequences, the players cannot be indifferent toward their outcome; players feel an urge to successfully respond to and solve the immediately pressing challenge. Therefore, computer gamers always show a strong disposition to solve situations arising in an episode. At the same time, it is uncertain whether the players will be able to solve the situation successfully or not. This state of uncertainty, combined with a strong wish to successfully achieve a goal, is the fundament of the experience of suspense. ${ }^{2,12}$ The resolution of the state of suspense at the end of an episode can, if the necessity to act has been successfully solved, lead to euphoric experiences of joy, and consequently result in video game enjoyment. ${ }^{2}$

\section{Hypothesis 5: The mechanism of suspense and solution is positively associated with video game enjoyment.}

Simulated experiences of life. On the third level of Klimmt's ${ }^{2}$ model, more complex and more comprehensive narrative structures that extend over several gaming sessions are involved. In the course of a video game, players aim at a main overarching goal, for example, saving the world. Gamers take on a specific role in trying to accomplish this goal, which is fundamentally different from their everyday experience. This kind of role taking in video games is suggested to be an enjoyment experience, because it provides relief by enabling the player to become immersed in an exciting fantasy world. Therefore, engaging in simulated experiences of life is expected to be related to enjoyment. ${ }^{2}$

Hypothesis 6: The mechanism of simulated experiences of life is positively associated with video game enjoyment. 


\section{Methods}

\section{Gaming activity}

In the present study, the game "The Elder Scrolls IV: OBLIVION" was used as a target game. "The Elder Scrolls" is a series of free role-playing games in which a maximum degree of freedom is given to players. Players can create their unique avatar by combining a plethora of available character attributes, and there is no fixed storyline in the games, which players are supposed to follow. Therefore, players can postpone or ignore the main quest for as long as they wish, while exploring the game world or following side quests.

\section{Participants}

The study involved 62 OBLIVION players aged between 13 and 54 years $(M=23.57, S D=7.50)$. The participants $(43$ male and 19 female) were recruited in the online-forum "gamestar.de" before release of the target game.

\section{Procedure}

Data were collected over a period of 9 weeks immediately following the release of "The Elder Scrolls IV: OBLIVION" in March 2006. During the period of data collection, participants accessed and completed a webbased questionnaire after each time they played OBLIVION to report on their gaming experiences. Participants played OBLIVION between 3 and 39 times during the period of data collection and accordingly answered the questionnaire between 3 and 39 times $\left(M_{\text {Number of sessions }}=13.26, S D=\right.$ 7.65). Totally, our participants reported on 822 gaming sessions. Detailed information on the distribution of the range of questionnaires is displayed in Figure 1.

The questionnaire consisted of both closed- and openended questions. First, participants answered a series of questions concerning the session, such as the date, time, and duration of the session. Second, participants were asked to report on the session in a short diary. Finally, participants answered a series of closed-ended questions on five- or sevenpoint Likert scales, presented in a random order.

\section{Measures}

Video game enjoyment. Video game enjoyment was measured by five items using a seven-point Likert scale (e.g., "I had a lot of fun during the session.") $(\alpha=0.961, M=5.12$, $S D=1.85)$.

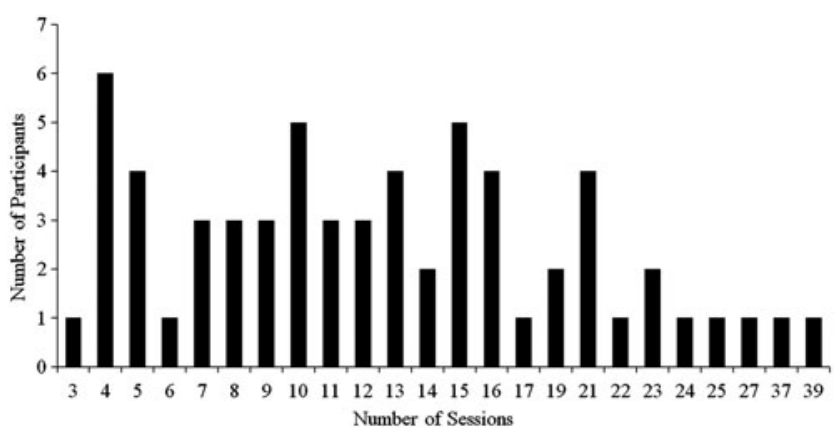

FIG. 1. Distribution of the range of questionnaires.
Exploratory behavior. Exploratory behavior was measured by a single item using a five-point Likert scale ("In this session I have explored new environments.") $(M=1.89$, $S D=1.17)$.

Involvement. Involvement in game tasks was measured by four items using a five-point Likert scale (e.g., "During the preceding session I was merged in my role as a hero in Tamriel." $)(\alpha=0.825, M=2.62, S D=0.99)$.

Spatial presence. Spatial presence was measured by four spatial presence self-location items from the MEC-SPQ ${ }^{13}$ using a five-point Likert scale (e.g., "It was as though my true location had shifted into the environment in the presentation.") $(\alpha=0.934, M=2.90, S D=1.16)$.

Competence. Perceived competence was measured by five items using a five-point Likert scale (e.g., "In this session I was able to successfully overcome challenges." $)(\alpha=0.754$, $M=2.26, S D=0.82$ ).

Breaks in self-efficacy. As the experience of self-efficacy is not cognitively accessible under normal conditions, it is not possible to measure it with closed-ended questions. ${ }^{2}$ Therefore, the responses to open-ended questions were coded to detect whether the experience of self-efficacy was interrupted during the sessions. Coding was executed in a dichotomous manner: if participants reported technical problems as a limitation of video game enjoyment, a break in self-efficacy was coded. If players did not mention technical problems, no break in self-efficacy was coded (Cohens's $\kappa=0.80, M=0.25$, $S D=0.43)$.

Suspense and solution. To measure whether players experienced suspense and solution as a source of enjoyment, they were asked to report on the extent to which coping with challenges contributed to their video game enjoyment, using a five-point Likert scale $(M=3.92, S D=1.31)$.

Simulated experiences of life. To find out whether simulated experiences of life played a role in evoking video game enjoyment, participants were asked to indicate the extent to which fulfilling their role in the video game contributed to their enjoyment, using a five-point Likert scale $(M=3.73$, $S D=1.27)$.

\section{Results}

A goal of the present study was to examine changes in video game enjoyment over time. Data were analyzed using a linear multilevel regression model, using SPSS v.19. In a multilevel analysis of data measured at several points in time, there is no requirement that (a) measurements begin and end at the same time for every individual in the study, (b) measurement intervals are equally spaced, or (c) the number of measurement points are the same between different individuals. ${ }^{14}$ Thus, multilevel regression modeling is an appropriate method for data analysis in this case, given the nature of our data. We set measurement occasions at the lowest level of analysis, with subjects as the grouping variable and used maximum likelihood estimation and a first-order autoregressive covariance matrix (AR1).

To investigate changes in video game enjoyment over time, we first applied a model in which only the accumulated 
gaming duration was used as a predictor variable. The results indicated that the gaming duration is a significant predictor of increased video game enjoyment over time (unstandardized regression coefficient $b=0.03, S E b<0.01$, $p<0.001)$.

Next, all predictor variables were included in the model. Again, the model generated a good fit (see Table 1). First, the model shows that the gaming duration is a significant predictor of changes in enjoyment $(b=0.08, S E b=0.01, p<0.001)$. We inserted a quadratic term for the gaming duration to check for improvements in the model. The quadratic term is a significant predictor of video game enjoyment $(b=-0.01, S E$ $b=0.01, p<0.01)$; the negative coefficient indicates that increases in video game enjoyment are diminished over time (the rate of increase decreases), at least for some participants.

Exploratory behavior was found to influence enjoyment; this variable interacts with involvement in the video game. The main effects of exploratory behavior can be interpreted because all of the measured variables represent grand meancentered values. Exploratory behavior was a significant predictor of video game enjoyment for all participants $(b=0.13$, $S E b=0.04, p<0.01)$. Therefore, Hypothesis 1a is supported. Furthermore, the expected interaction between exploratory behavior and involvement was significant $(b=-0.09, S E$ $b=0.04, p<0.05)$, indicating that exploring the environment enhances video game enjoyment, especially for those players with low involvement in game tasks (Fig. 2). This finding is consistent with Hypothesis $1 \mathrm{~b}$.

As predicted by Hypothesis 2, the experience of spatial presence is significantly related to video game enjoyment ( $b=0.24, S E b=0.08, p<0.01)$. Furthermore, the experience of competence during gaming sessions is significantly related to an increase in video game enjoyment $(b=0.19, S E b=0.08$, $p<0.05)$. Therefore, the data also support Hypothesis 3.

Breaks in self-efficacy were not significantly related to enjoyment ( $b=-0.13, S E b=0.10, n s$, not significant); therefore, Hypothesis 4 was not confirmed. As predicted by Hypothesis 5 , experiences of suspense and solution are a significant predictor of changes in video game enjoyment $(b=0.18, S E$ $b=0.05, p<0.01)$. In addition, engagement in simulated life experiences contributes significantly to the experience of video game enjoyment $(b=0.15, S E \quad b=0.05, p<0.001)$. Therefore, Hypothesis 6 is supported.

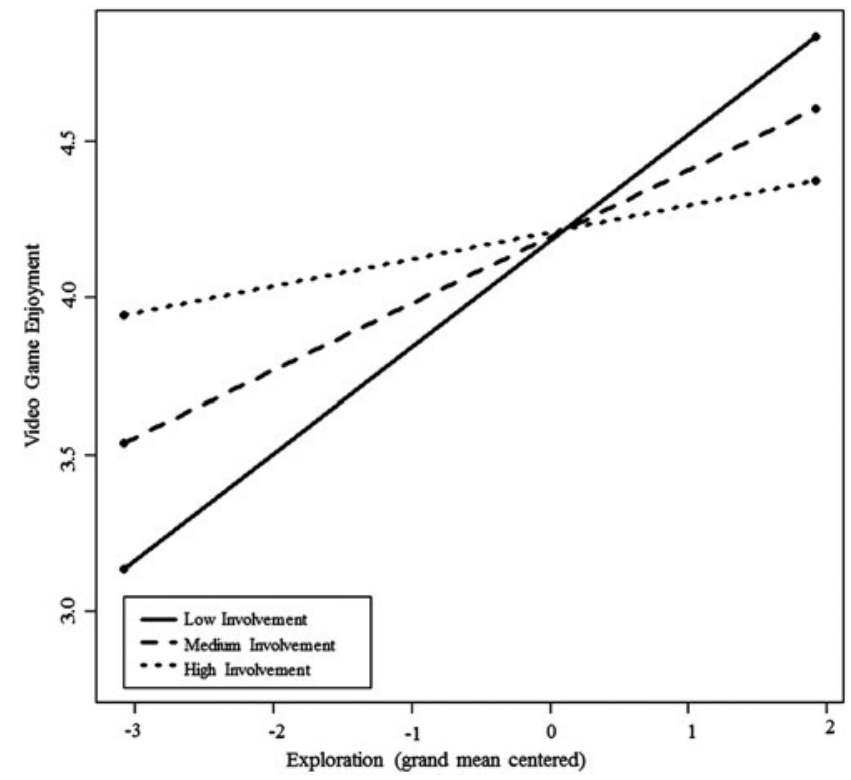

FIG. 2. Interaction between exploratory behavior and involvement in game tasks.

\section{Discussion}

First, the dynamic approach used in the present study allows conclusions about changes in video game enjoyment over relatively long time periods. The results of the present study indicate that game playing over several sessions leads to an increase in video game enjoyment, as represented by both linear and quadratic trends. However, it appears that the generally increasing trend in enjoyment is moderated over time, at least for some players.

Second, the present study is the first to show a statistically significant correlation between video game enjoyment and the variable "simulated experiences of life", which considers the narrative content that extends beyond a single gaming session. Also, the present study is the first to empirically verify the interaction between exploratory behavior and involvement in game tasks.

Fourth, our approach of incorporating several predictor variables for video game enjoyment into one model and

Table 1. Results of a Multilevel Model Growth Analysis of Predictor Variables as a Function of Video Game Enjoyment

\begin{tabular}{lrrrr}
\hline & $\mathrm{b}$ & $\mathrm{SE} \mathrm{b}$ & 95 percent CI & $\mathrm{p}$-Value \\
\hline Constant term & 2.67 & 0.31 & $2.06,3.29$ & 0.000 \\
Gaming duration & 0.08 & 0.01 & $0.05,0.10$ & 0.000 \\
Gaming duration $\times$ gaming duration & -0.01 & 0.01 & $-0.02,-0.00$ & 0.001 \\
Exploratory behavior & 0.13 & 0.04 & $0.06,0.22$ & 0.001 \\
Exploratory behavior $\times$ involvement & -0.09 & 0.04 & $-0.16,-0.02$ & 0.018 \\
Spatial presence & 0.24 & 0.08 & $0.09,0.39$ & 0.001 \\
Competence & 0.19 & 0.08 & $-0.33,0.07$ & 0.020 \\
Breaks in self-efficacy & -0.13 & 0.10 & $0.09,0.28$ & 0.196 \\
Suspense and solution & 0.18 & 0.05 & $0.04,0.25$ & 0.006 \\
Simulated experiences of life & 0.15 & 0.05 & 0.000 \\
\hline
\end{tabular}

$b$, slope of the regression (except for the constant term); SE b, standard error of the slope; 95 percent CI, confidence interval of the slope; $p$-value, probability that the slope value is 0 . 
collecting the data in real-world conditions was successful. We showed that the variables that influence enjoyment in cross-sectional settings are also relevant to explanations of long-term changes in video game enjoyment. Moreover, the results of the present study are in accordance with research by theorists describing player motivations. For example, the predictor variable exploratory behavior is closely related to the motivational subcomponent "Discovery" described by Bartle, ${ }^{15}$ whereas perceived competence appears to be the psychological process behind the "Achievement"-Motivation $\mathrm{Yee}^{16}$ revealed. The results of the present study are, therefore, an important contribution toward bringing video game research from the laboratory into the field.

\section{Study Limitations}

In terms of limitations, the independent variable that did not predict video game enjoyment as expected, breaks in selfefficacy, should be considered. The absence of a significant relationship between breaks in self-efficacy and video game enjoyment may be an artifact of the method used to measure breaks in self-efficacy. Participants may have reported technical problems as a temporary limitation of their experience of enjoyment, but did nevertheless report an increase in the enjoyment in the session as a whole. Therefore, breaks in selfefficacy may not have shown the expected effect on video game enjoyment. Future research should strive to establish a valid operationalization of that parameter.

Moreover, the results discussed in the present article are constrained to one game type and it is not clear whether the effects noted in the present study can be extended to other video game genres. Modern role-playing games may feature specific characteristics that foster different kinds of associations between the included predictor variables and video game enjoyment. For example, it would be interesting to examine whether feelings of spatial presence contribute to the experience of video game enjoyment in video games that do not use a first person viewpoint or similar presence-inducing attributes. However, future research should explore the effects observed in the present study in a variety of video games and video game genres.

\section{Author Disclosure Statement}

No competing financial interests exist. This manuscript has not been published nor is it under consideration for publication elsewhere.

\section{References}

1. Behr KM, Klimmt C, Vorderer P. (2009) Leistungshandlen und Unterhaltungserleben im Computerspiel. In Quandt T, Wimmer J, Wolling J, eds. Die Computerspieler. Studien zur Nutzung von Computergames. Wiesbaden: VS Verlag für Sozialwissenschaften, pp. 225-240.

2. Klimmt C. (2006) Computerspielen als Handlung. Dimensionen und Determinanten des Erlebens interaktiver Unterhaltungsangebote. Köln: Herbert von Halem Verlag.
3. Klimmt C, Hartmann T, Frey A. Effectance and control as determinants of video game enjoyment. CyberPsychology and Behavior 2007; 10:845-847.

4. Klimmt C, Blake C, Hefner D, et al. (2009) Player performance, satisfaction, and video game enjoyment. In Natkin $S$, Dupire J, eds. Eighth International Conference on Entertainment Computing 2009, Lecture Notes in Computer Science 5709. Berlin: Springer, pp. 1-12.

5. Vorderer P, Hartmann T, Klimmt C. (2003) Explaining the enjoyment of playing video games: the role of competition. In Marinelli D, ed. Proceedings of the 2nd International Conference on Entertainment Computing (ICEC 2003), Pittsburgh, NY: ACM, pp. 1-8.

6. Wirth W, Hartmann T, Böcking S, et al. A process model of the formation of spatial presence experiences. Media Psychology 2007; 9:493-525.

7. Lombard M, Ditton T. At the heart of it all: the concept of presence. Journal of Computer-Mediated Communication 1997; 3. http://jcmc.indiana.edu/vol3/issue2/lombard.html (accessed July 26, 2011).

8. Sylaiou S, Mania K, Karoulis A, et al. Exploring the relationship between presence and enjoyment in a virtual museum. International Journal of Human-Computer Studies 2010; 68:243-253.

9. Tamborini R, Bowman ND. (2010) Presence in video games. In Campanella Bracken C, Skalski PD, eds. Immersed in media. Telepresence in everyday life. New York: Routledge, pp. $87-112$.

10. Gee JP. What video games have to teach us about learning and literacy. Computers in Entertainment 2003; 1:1-4.

11. Tamborini R, Bowman N, Eden A, et al. Defining media enjoyment as the satisfaction of intrinsic needs. Journal of Communication 2010; 60:758-777.

12. Zillmann D (1996) The psychology of suspense in dramatic exposition. In Vorderer P, Wulff HJ, Friedrichsen M, eds. Suspense: conceptualizations, theoretical analyses, and empirical explorations. Mahwah: Lawrence Erlbaum Associates, pp. 199-231.

13. Vorderer P, Klimmt C, Ritterfeld U. Enjoyment: at the heart of media entertainment. Communication Theory 2004; 14: 388-408.

14. Hayes A. A primer on multilevel modelling. Human Communication Research 2006; 32:385-410.

15. Bartle R. (2004) Designing virtual worlds. Berkeley: New Riders.

16. Yee N. Motivations for play in online games. CyberPsychology and Behavior 2006; 9:772-775.

Address correspondence to: Fabian Ryffel Institute of Mass Communication and Media Research University of Zurich Andreasstrasse 15 Zurich 8050 Switzerland

E-mail: f.ryffel@ipmz.uzh.ch 